\title{
OPEN Creating hot spots within air for better sensitivity through design of oblique-wire-bundle metamaterial perfect absorbers
}

\author{
Xin-Xian Wu, Cheng-Yu Lu \& Tsung-Yu Huang ${ }^{\bowtie}$
}

Better sensitivity of a biosensor could boost up the detection limit of analytes, thus a must in the fields of bio-sensing and bio-detection. To further enhance the sensitivity of a biosensor, in this work, we design an oblique-flat-sheet metamaterial perfect absorber (MPA) to concentrate the hot spots within air between the oblique flat sheet and the continuous ground metal, thus enabling fully interaction between analytes and hot spots. The corresponding field distributions in simulation corroborated our assumption and its sensitivity could be up to $1049 \mathrm{~nm} / \mathrm{RIU}$. Then, we fabricated the sample by e-beam lithography process for a seed layer and simply tilting the sample during deposition to obtain oblique flat sheets. When considering the stochastic nature of the deposited multiple oblique flat sheets, we modified the metallic upper resonator of the MPA from the single oblique-flat-sheet into randomly distributed oblique-wire-bundle (OWB) and in simulation, its sensitivity is boosted up to $3319 \mathrm{~nm} / \mathrm{RIU}$. In experiments, the measured sensitivity is $1329 \mathrm{~nm} / \mathrm{RIU}$ under different concentrations of glucose solutions that is four times larger than the $330 \mathrm{~nm} / \mathrm{RIU}$ of the planar MPA. The higher sensitivity was attributed to that the OWB MPA could provide hot spots within air not only between OWB and grounded metal but also among wires. Moreover, the OWB could also trap and concentrate the analytes locally.

Since metamaterials proposed by Pendry and Smith et al. emerged in $1999^{1,2}$, within these two decades, they became phenomenal success in different kinds of meta-devices and are widely applied in different fields, for example, invisibility cloaks for stealthy technology $y^{3,4}$, slow light for optical storage and computation ${ }^{5-7}$, metamaterialbased sensors for bio-sensing/chemical detection ${ }^{8-10}$. Among these meta-devices, metamaterial-based sensors are one of the most important developing devices due to the strong requirement for food safety detection ${ }^{11-13}$ and explosive detection ${ }^{14}$ and have attracted many researchers' efforts on further improving their sensitivity for lower detection limit. When employed in the design of a sensor, metamaterials could provide concentrated electric fields adjacent to resonators, also known as hot spots; when analytes interact with these hot spots, the resonating frequency of metamaterials would red-shift, enabling the detection and analysis of the analytes. Based on this mechanism, to further enhance the sensitivity of metamaterial-based sensors, researchers tried to generate a very strong hot spot by different methods such as different resonator designs ${ }^{8,15,16}$, plasmonic nanoparticles ${ }^{17,18}$, gap plasmon ${ }^{19-21}$, and metamaterial perfect absorbers ${ }^{8,10,15,22}$. Plasmonic nanoparticles such as gold or silver nanoparticles obtained from chemical synthesis could provide ultra-strong electromagnetic field enhancement and large-area hot spots due to their small gaps among particles. Besides, the synthesis of nanoparticles is low cost and easy. On the other hand, the gap plasmons excited from the two adjacent metallic resonators also provide large field enhancement within the precisely controlled small gaps. Finally, metamaterial perfect absorbers consisted of a sandwiched metal-dielectric-metal structure concentrate the field at the dielectric spacer, thus also offering a strong hot spot. Nevertheless, the nanoparticles would suffer from the insufficiencies of random distribution of the particles, thus resulting in poor stability and repeatability and the gap plasmons also showed the disadvantages including small-area hot spots and less interaction between analytes and hot spots. It is the metamaterial perfect absorbers, one of the most promising candidates to achieve higher sensitivity. 

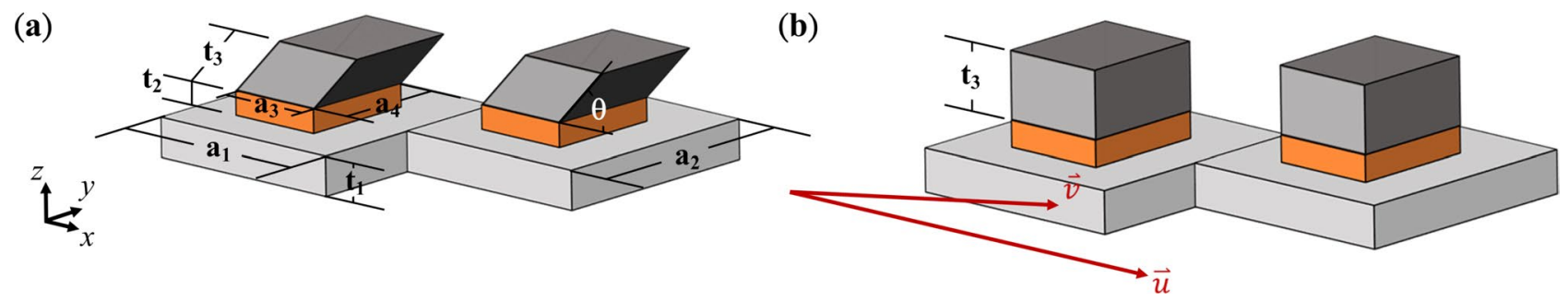

Figure 1. Schemes of (a) oblique-flat-sheet (OFS) and (b) planar metamaterial perfect absorbers. Here, $a_{1}$ is $800 \mathrm{~nm}, \mathrm{a}_{2} 850 \mathrm{~nm}, \mathrm{a}_{3} 45 \underline{0} \mathrm{~nm}, \mathrm{a}_{4} 700 \mathrm{~nm}, \mathrm{t}_{1} 150 \mathrm{~nm}, \mathrm{t}_{2} 120 \mathrm{~nm}, \theta$ 40-degree and $\mathrm{t}_{3} 455 \mathrm{~nm}$. Meanwhile, $\vec{u}=2 \vec{x}$ and $\vec{v}=\vec{x}+0.5 \vec{y}$.

Still, the hot spot would concentrate within the dielectric layers of the MPAs, thus hindering the full interaction between analytes and hot spots. The sensitivity of the planar MPAs are ranged around 225-1000 nm/ $\mathrm{RIU}^{8,23,24}$; to solve this issue, some researchers proposed three dimensional metamaterials that provide a hot spot within air ${ }^{22,25-31}$; alternatively, researchers proposed a micro/nano-fluidic integrated MPA to locate the hots spots within the fluidic channel where analytes could fully interact with hot spots ${ }^{32-35}$. The authors also employed Fano-resonance induced by coupling between molecular absorption and resonance absorption from metamaterials to enhance its sensitivity and detection limit ${ }^{35,36}$. Moreover, Su et al. proposed a vertical-wall MPA based on novel e-beam lithography procedure, plasma-etching process and oblique deposition method ${ }^{37}$. The verticalwall MPA is composed of gold/air/gold three-layered structure. The air channel could facilitate full interaction between hot spots and analytes, thus enhancing its sensitivity. Nonetheless, all the above-mentioned methods inevitably increased the fabrication difficulties which might lower the yield and stabilities of the devices. Also, the sensitivities are only a little bit better than or comparable to the planar MPAs, for example, 885/900 nm/RIU in Refs. ${ }^{22}$ and $^{27}$ for 3D metamaterials, 3.5 THz/RIU at the frequency of $6.4 \mathrm{THz}$ and $140 \mathrm{GHz} / \mathrm{THz}$ at $\sim 0.8 \mathrm{THz}$ in Refs. ${ }^{32}$ and $^{33}$ for micro/nano fluidic MPAs. Therefore, in this work, we proposed a simple method to enhancing the sensitivity of a metamaterial-based sensor by tilting the substrate to a certain angle to conduct oblique deposition ${ }^{38-42}$ with a periodic seed layer fabricated by e-beam lithography process and results in an oblique-flatsheet (OFS) metamaterial perfect absorber. The field would concentrate between the oblique sheet and ground, thus providing space for analytes to fully interact with the hot spots.

\section{Design and simulation}

To achieve a unity absorption, the OFS MPA should satisfy the condition of destructive interference governed by $\tilde{r}=\tilde{r}_{12}-\frac{\tilde{t}_{12} \tilde{t}_{21} e^{i 2 \beta}}{1+\tilde{r}_{21} e^{i 2 \beta}}$, whereas $\tilde{r}$ is the total reflection, $\tilde{r}_{12}, \tilde{r}_{21}, \tilde{t}_{12}$ and $\tilde{t}_{21}$ are reflection and transmission coefficients at the interface from the upper resonator to air and from air to the upper resonator, respectively. $\beta$ is the propagation constant within the dielectric layer of the $\mathrm{MPA}^{43,44}$. Once $\tilde{r}$ is 0 , we could achieve perfect absorption. To design the OFS MPA, all the dimensions should be chosen to meet the requirement for a guarantee of occurrence of the shadowing effect during oblique deposition and for an absorption band that is free of water absorption. Thus, we should first determine dimensions of the seed layer for the deposition of the oblique flat sheets. Here, to avoid residual metal deposition at the positions where no seed layers exist, unlike the conventional rectangular metamaterial array, we employed a parallelogram array with translation vectors of $\overrightarrow{\boldsymbol{u}}$ and $\overrightarrow{\boldsymbol{v}}$ equal to $2 \vec{x}$ and $\vec{x}+0.5 \vec{y}$, respectively. Then, to confine all the deposited metal only on top of the seed layer, a coverage length for self-shadowing effect could be calculated by Eq. $(1)^{40}$ shown below

$$
\mathrm{s}=\mathrm{h} \times \tan (\alpha)
$$

where s denotes the coverage length of the self-shadowing effect, $h$ the thickness of the seed layer and $\alpha$ the inclination angle of the substrate. Note that s should be always larger than the translational vector $\overrightarrow{\boldsymbol{u}}$ along the $\mathrm{x}$-direction of the MPA to guarantee no metal would grow outside the seed layer. Next, the inclination angle $\alpha$ would influence the maximum achievable angle $\beta$, the angle between the oblique flat sheet and grounded metal's normal; such angles vary with different choices of metal. In this work, as shown in Fig. 1a, the periodicities of the MPA along the $\mathrm{x}$ - and y-direction are 800 and $850 \mathrm{~nm}$, respectively. The thickness of grounded metal is $150 \mathrm{~nm}$ and the length, width and thickness of the seed layer are 455, 700 and $120 \mathrm{~nm}$, respectively. The maximum angle $\beta$ is 50-degree obtained from the experiments while $\alpha$ is 86 -degree; thus, in simulation, the angle $\theta$ between the oblique flat sheet and the substrate (i.e., $\theta=90-\beta$ ) is set to 40 degree. The seed layer is composed of $\mathrm{MgF}_{2}$ with permittivity of 1.8868 and loss tangent of 0.01 . It is worth mentioning that the calculated coverage range for the parameter is $1716 \mathrm{~nm}$, which is larger than $1600 \mathrm{~nm}$ and guarantees limited metal deposition only on top of the seed layer. Besides, the side-length of the oblique-flat-sheet is $455 \mathrm{~nm}$. Finally, to examine the effect of sensitivity enhancement from our proposed method, we also simulate a planar MPA with the same parameters as the OFS MPA in order to make a fair comparison as shown in Fig. 1b. Note that we employed CST Microwave Studio with an algorithm of finite integration method and in simulation, the boundary conditions we employed are open and unit cell parallel and perpendicular to the propagation direction, respectively.

The absorbance spectra of the OFS MPA and planar MPA are depicted in Fig. 2a, b, respectively; there appear significant absorbance peaks at the frequencies of 201.69 and $205.43 \mathrm{THz}$ with a magnitude of $94.57 \%$ and $78.16 \%$, respectively. Then, we applied analytes on the MPAs with the refractive index changing from 1 to 2 

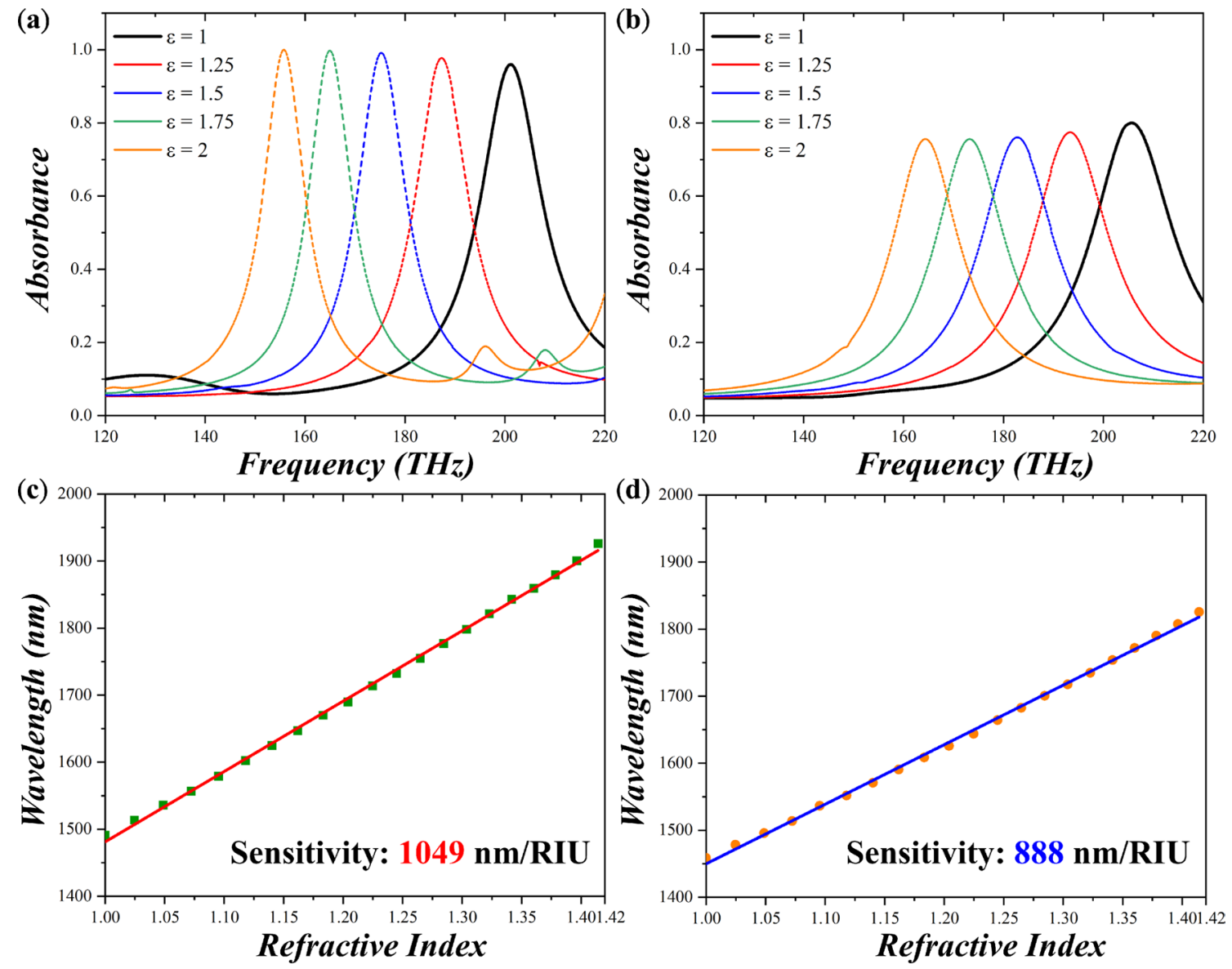

Figure 2. Absorbance of (a) the OFS and (b) the planar MPAs under environments with different refractive indices from 1 to 2 . Resonant wavelengths with respect to the refractive index for (c) the OFS and (d) the planar MPAs. The corresponding sensitivity is 1049 and $888 \mathrm{~nm} / \mathrm{RIU}$, respectively.
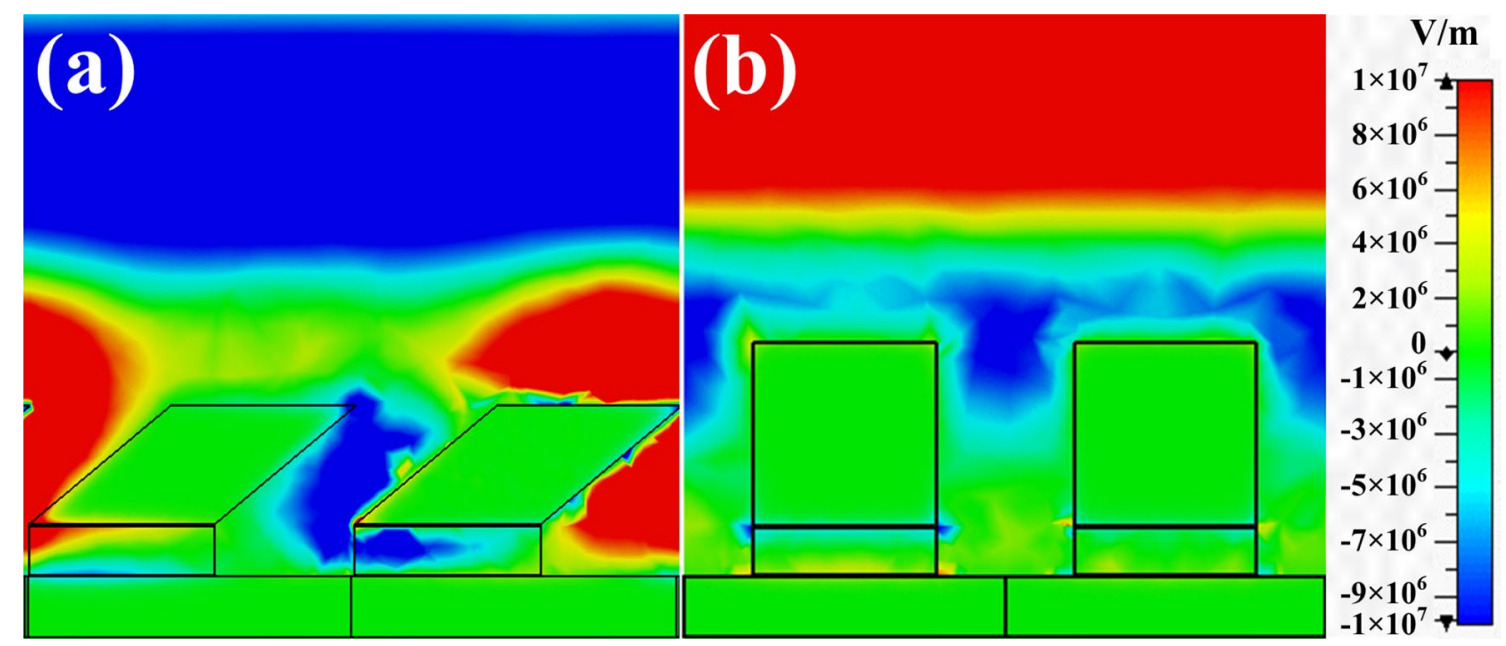

Figure 3. Field distributions of (a) the OFS MPA at 201.69 THz and (b) the planar MPA at 205.43 THz. The OFS MPA revealed stronger electric field and larger area hot spots compared to the ones of planar MPA.

and the absorbance peaks of the both MPAs are red-shifted. The sensitivity ( $\mathrm{S}=\Delta \lambda / \Delta n$ where $\Delta \lambda$ is resonance wavelength change while $\Delta n$ is refractive index change) of the OFS MPA is $1049 \mathrm{~nm} / \mathrm{RIU}$ that is higher than $888 \mathrm{~nm} / \mathrm{RIU}$ of the planar MPA as depicted in Fig. 2c, d. It is 1.18 folded enhancement compared to the planar MPA. Taking a step further, we calculated the figure of merit (FoM $\left.=\frac{\text { Sensitivity }}{\text { Bandwidth }}\right)$ for the both MPAs. The FoM for the OFS and planar MPAs are 9.52 and 7.90. The higher sensitivity and larger FoM reveal the superior sensing behavior of our proposed OFS MPA compared to the planar one. To further dig into the hidden mechanism, 

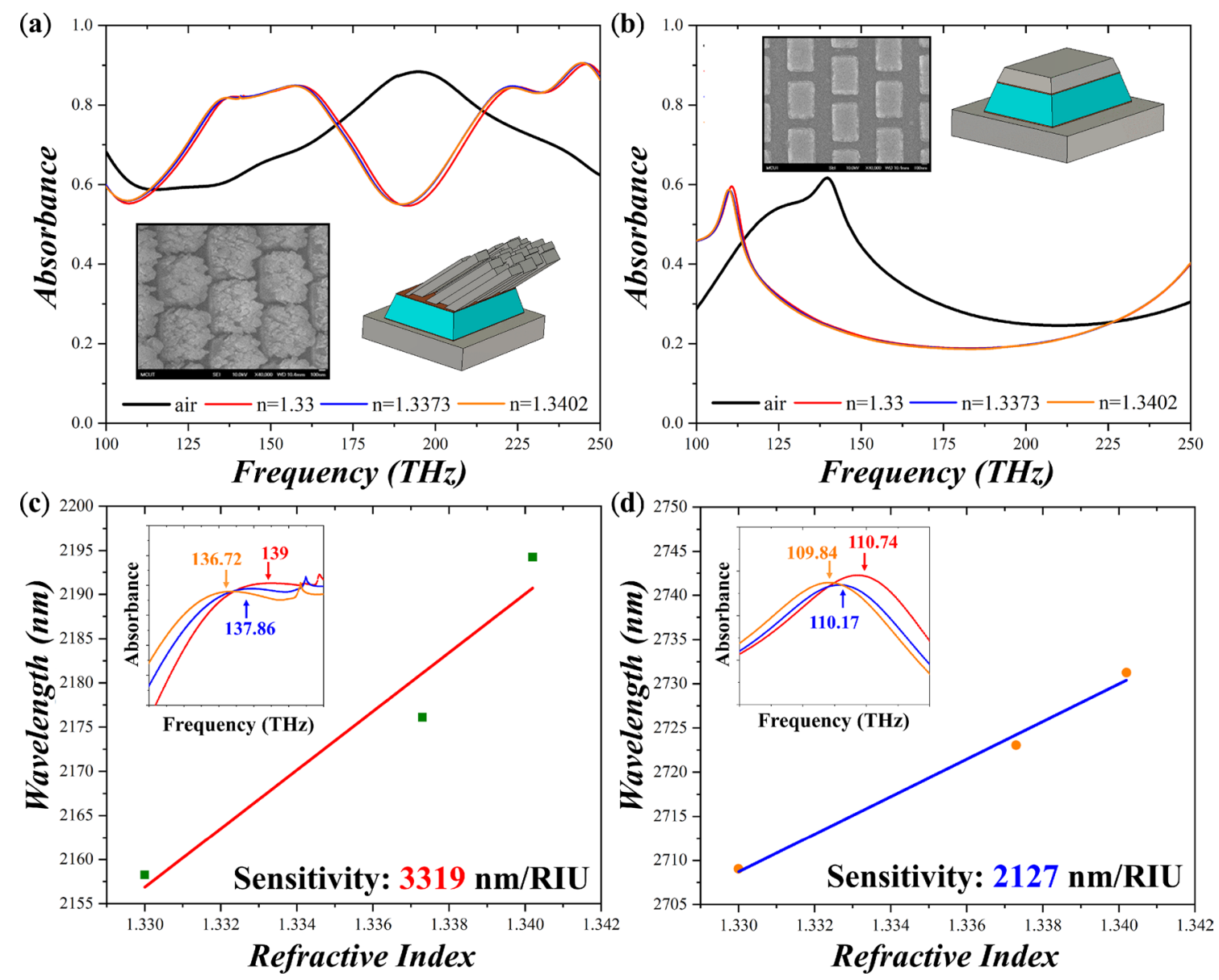

Figure 4. Absorbance of (a) the oblique-wire-bundle (OWB) and (b) the planar MPAs under ambient environments and different concentrations of glucose solutions with refractive index from 1.33 to 1.3402 . Insets depict SEM images of the as-fabricated samples and their modified morphologies in simulation. Resonant wavelengths with respect to the refractive index for (c) the OWB and (d) the planar MPAs. The corresponding sensitivity is 3319 and $2127 \mathrm{~nm} / \mathrm{RIU}$, respectively. Insets show enlarged spectra near resonant frequencies.

we monitored the electric field distribution at the frequency of the absorbance peaks. From Fig. 3a, b, we clearly observe that for the OFS MPA, the hot spots indeed located within the air regime between the oblique flat sheet and grounded metal, thus fully interacting with analytes and resulting in better sensitivity. In contrast, the hot spots of the planar MPA concentrated on the dielectric layers, providing limited interaction with analytes and thus smaller sensitivity.

\section{Fabrication and measurement}

To fabricate the sample, we conducted electron beam vapor deposition and e-beam lithography for deposition and patterning, respectively to make a periodic array to mimic the unit cell boundary condition in simulation (see "Methods" section for details). Then, the sample was put on a holder that was tilted an angle of 86-degree to the horizon for oblique deposition. To compensate potential manual error when putting the sample on the holder, we increased the thickness of the seed layer up to $166 \mathrm{~nm}\left(160 \mathrm{~nm} \mathrm{MgF}_{2}\right.$ and $\left.6 \mathrm{~nm} \mathrm{Ti}\right)$, thus guaranteeing a coverage length of $2374 \mathrm{~nm}$. SEM images of the as-fabricated sample are illustrated in the inset of Fig. 4. Compared to the simulation, the as-fabricated sample revealed a stochastic distribution of nano-wire bundles on top of the seed layers. Also, the dimensions deviate to the simulated one because of the inevitable fabrication errors from the lithography process. Furthermore, due to undercut of the resist, the seed layer, instead of a cuboid, became a frustum of a rectangular pyramid with a tilted angle of 16-degree of the side length. To reflect the morphology of the fabricated sample, in simulation, the periodicities along $\mathrm{x}$ - and y-direction became $795 \mathrm{~nm}$ and $855 \mathrm{~nm}$; the length and width of the seed layer were 550 and $740 \mathrm{~nm}$. The side length of the oblique wire bundles is around 290-350 nm with a random distribution. On the other hand, the planar MPA also shows some dimensional deviation including the periodicities of 810 and $855 \mathrm{~nm}$ along $\mathrm{x}$ - and $\mathrm{y}$-direction, the length of 480 and width of 700 for the seed layer. Here, the seed layer also became a frustum of a rectangular pyramid with a tilted angle of 16-degree of the side length. In addition, due to the fabrication difficulty, we reduced the thickness of the upper metal down to $80 \mathrm{~nm}$. Figure $4 \mathrm{a}$, b shows the simulated absorbance for the OWB and planar MPA, respectively (see Supplementary Materials for polarization and incident angle dependence of the OWB MPA). There appears an absorbance peak at $195.18 \mathrm{THz}$ for the OWB MPA and at $138.96 \mathrm{THz}$ for the planar MPA. Here, we could 


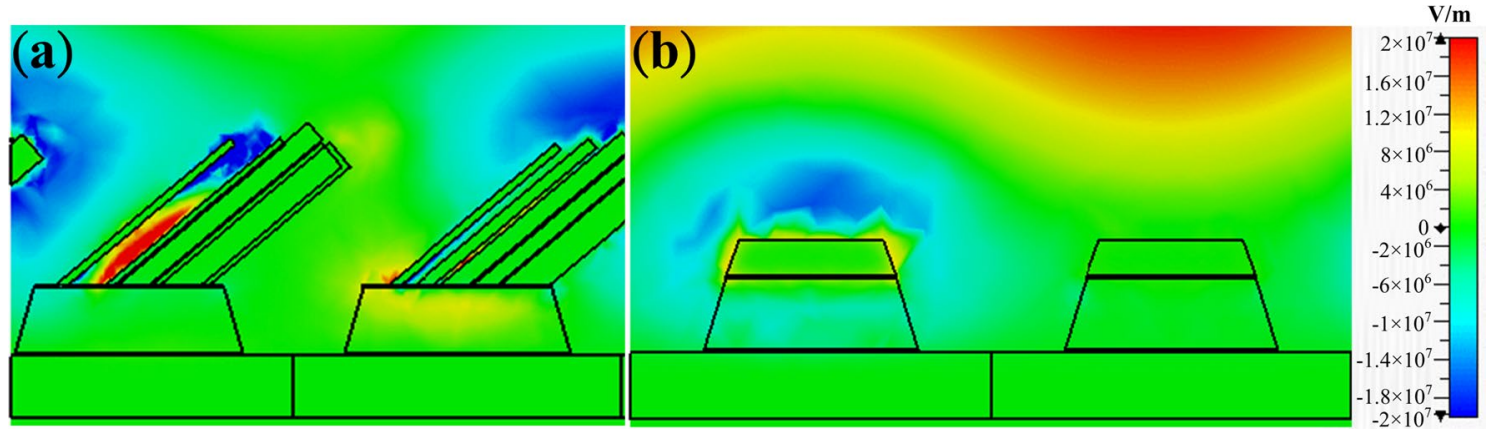

Figure 5. Field distributions of (a) the OWB MPA at $195.18 \mathrm{THz}$ and (b) the planar MPA at $138.96 \mathrm{THz}$. The OWB MPA revealed stronger electric field and larger area hot spots compared to the ones of planar MPA. Note that the gaps among nanowires also provided hot spots, thus further enhancing the sensitivity of the MPA.

observe that the OWB MPA revealed broader absorption bandwidth compared to the planar MPA. Such broad absorption could be attributed to two factors. One is that since OWB MPA possessed many wires instead of a single flat sheet and each wire could contribute absorption at different but similar frequencies, thus the OWB MPA showed broad absorption bandwidth. The other is that the coupling among wires might also contribute to the broadening of absorption bands ${ }^{42}$. Besides, although the OWB MPA is asymmetric, still the cross-polarization reflectance is small $(\sim 4 \%)$ compared to co-polarization reflectance. Here, we further simulated the frequency change with the addition of deionized water and glucose solutions. The refractive indices of the deionized water and $3 \%$ and $5 \%$ glucose solutions are $1.33,1.3373$ and 1.3402 , respectively. The corresponding sensitivity is $3319 \mathrm{~nm} /$ RIU and $2127 \mathrm{~nm} /$ RIU for the OWB and planar MPAs as illustrated in Fig. 4c, d. The sensitivity of the OWB MPA is 1.56 times the value of the planar MPA, evidencing the usefulness of the proposed method for sensitivity enhancement. Note that since the lower resonance frequency guaranteed better sensitivity, thus the enhancement of sensitivity could be larger from the OWB MPA if the resonating frequencies of the two are the same ${ }^{10}$. Again, the corresponding field distributions of the OWB and planar MPAs are recorded and plotted in Fig. 5a, b at the frequencies of 195.18 and $138.96 \mathrm{THz}$. Overall, the field of the planar MPA is much weaker compared to the one of the OWB MPA. Also, the OWB MPA could provide the larger area of the hot spots. Further, the fields concentrate not only between the oblique wires and grounded metal plane but also among nanowires, providing much more hot spots than the OFS MPA.

To measure absorbance of the fabricated MPAs, we employed $\mu$-Fourier-transformed infrared spectroscopy (Bruker V70 equipped with a microscope), the light spot was focused onto the MPA area equal to $100 \times 100 \mu \mathrm{m}^{2}$. The measured absorbance spectra are illustrated in Fig. 6a, b for the OWB and planar MPAs. The simulated spectra are also included. The first two absorbance peaks of the OWB MPA correspond to the simulated results well and so does the absorbance peak of the planar MPA. Still, some deviations such as smaller absorbance of the two peaks and larger absorbance for the gap between the two peaks for the OWB MPA and minor absorbance peaks for the planar MPA. Such deviation could be attributed to the different permittivity dispersion and dimensional variation in simulation and in experiments. Next, we used deionized water, and $3 \%$ and $5 \%$ glucose solutions as the analytes to test the sensitivity of the two MPAs. By recording its resonance wavelength change with respect to the refractive index change under different concentrations of glucose solutions, we can retrieve the sensitivity in experiments. Unfortunately, due to strong water absorption from water, only frequency shifts around $140 \mathrm{THz}$ could be observed. The corresponding sensitivity of the OWB MPA is $1329 \mathrm{~nm} / \mathrm{RIU}$ which is 4 times larger than $330 \mathrm{~nm} / \mathrm{RIU}$ of the planar MPA as shown in Fig. 6c, d, respectively. Such difference could be related to the location and the area of the hot spots. It is worth mentioning that the smaller sensitivity in experiments than in simulation might originate from the fabrication error and high losses from metal by oblique deposition method. Moreover, when scrutinizing the simulated and measured sensitivity, the measured sensitivity of the planar MPA degraded much severely compared to the one of the OWB MPA. Such behavior might originate from that in experiments, due to stochastically distributed oblique wires, the structure could provide a trap for the analytes and concentrate the analytes locally. This locally higher concentration would induce larger frequency shift and so does the sensitivity. On the contrary, the planar MPA cannot trap analytes, thus showing a significant degradation of the sensitivity. Finally, we tabulated the sensitivity of different metamaterial perfect absorbers in Table 1 for comparison. In this summary, our proposed OWB MPA possessed higher sensitivity not only in simulation but also in experiments.

\section{Conclusions}

With the abovementioned simulation and measurement results, we designed, and simulated the oblique-flatsheet metamaterial perfect absorber. The OFS MPA shows sensitivity of $1049 \mathrm{~nm} / \mathrm{RIU}$. Then, we conducted e-beam deposition and lithography procedure to fabricate the sample. To take the dimensional change of the as-fabricated sample into consideration, in simulation, we amended the structure into a stochastically-distributed oblique-wire-bundle MPA based on the SEM image and such modifications resulted in sensitivity up to $3319 \mathrm{~nm} /$ RIU, almost 3.2 folded enhancement. In addition, the sample was characterized by $\mu$-FTIR and revealed a good agreement between the measured and simulated spectra. Finally, to test the experimental sensitivity, deionized water, and $3 \%$ and $5 \%$ glucose solutions were applied onto the sample. The sensitivity is $1329 \mathrm{~nm} / \mathrm{RIU}$ which is 
(a)
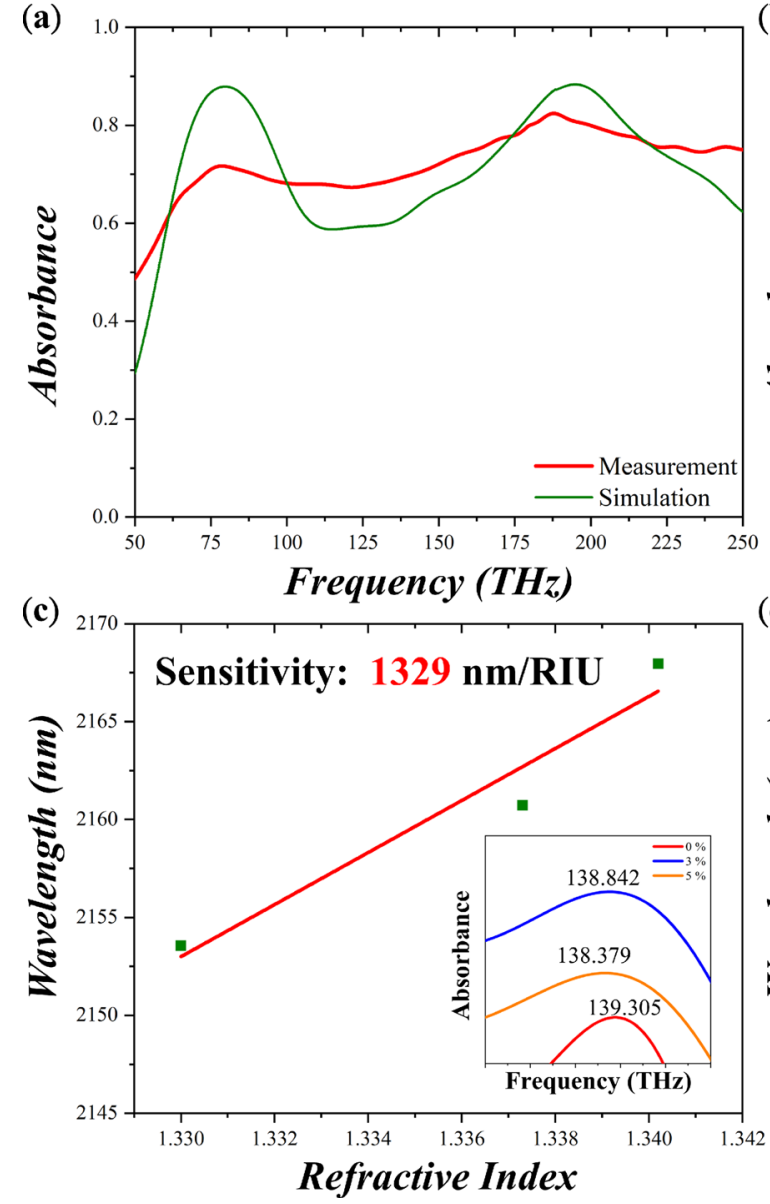
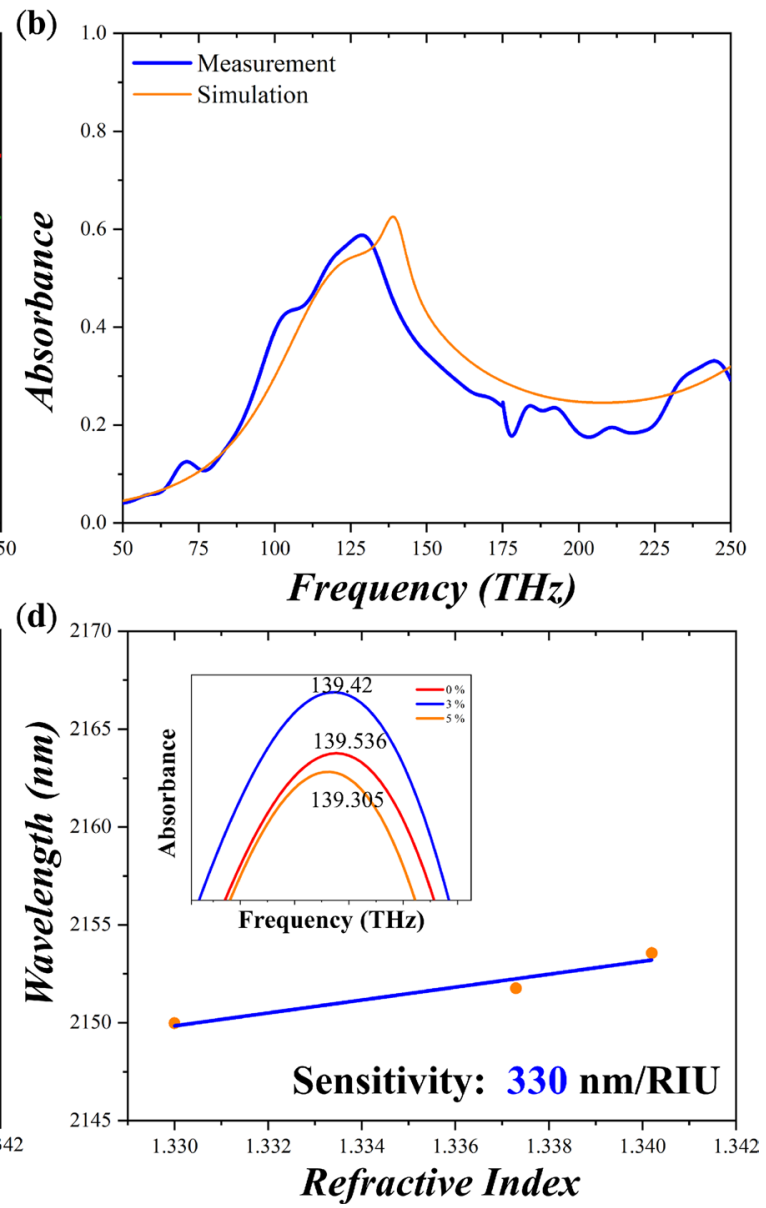

Figure 6. Measured absorbance spectrum of (a) the OWB MPA and (b) the planar MPA. Measured results agree the simulated ones well. The resonant wavelength with respect to refractive index for (c) the OWB MPA and (d) the planar MPA with the sensitivity of $1329 \mathrm{~nm} / \mathrm{RIU}$ and $330 \mathrm{~nm} / \mathrm{RIU}$, respectively. Insets depict the enlarged spectra near the resonance frequency.

\begin{tabular}{|l|l|l|l|}
\hline Work & Upper resonator & Operating frequency (THz) & Sensitivity (nm/RIU) \\
\hline Our work & Oblique-wire-bundle & 139 & $\begin{array}{l}3319 \\
1329^{*}\end{array}$ \\
\hline Ref. $^{45}$ & Planar ring/disk & 150 & 225 \\
\hline Ref. $^{23}$ & Split-rings & 193 & 500 \\
\hline Ref. $^{24}$ & C-shaped spit-ring with a thin wire & 390 & 1000 \\
\hline Ref. $^{46}$ & Suspended microracetrack & 150 & 337.5 \\
\hline Ref. $^{47}$ & Triangle cavity coupled with ellipse-ring & 350 & 860 \\
\hline Ref. $^{48}$ & Planar square array & 236 & 840 \\
\hline Ref. $^{29}$ & 3D split ring & 280 & 300 \\
\hline Ref. $^{31}$ & 3D Gold disk with aluminum pillars & 556 & $683.5^{*}$ \\
\hline
\end{tabular}

Table 1. Comparison of the sensitivity of metamaterial perfect absorbers. Note that * indicated experimentally measured sensitivity.

4 times larger than $330 \mathrm{~nm} / \mathrm{RIU}$ of the planar MPA. To conclude, we provide, in this work, a simple method, by just tilting the sample an angle with respect to the horizon, to boosting the sensitivity of the MPA-based sensors. We believe this work would pave the route toward future advanced bio-sensing and bio-imaging.

\section{Method}

E-beam lithography and deposition procedure. To construct an OWB MPA, a silicon substrate was cleaned on which 150-nm-thick aluminum was deposited. Then, A5 PMMA resist was spin-coated with a rotation speed of $1000 \mathrm{rpm}$ for $10 \mathrm{~s}$ and then a rotation speed of $4000 \mathrm{rpm}$ for $60 \mathrm{~s}$. Prebake the sample up to $180{ }^{\circ} \mathrm{C}$ 
for $3 \mathrm{~min}$. Next, the sample was exposed to electrons with a dosage of $200 \mu \mathrm{C} / \mathrm{cm}^{2}$ and then immersed in the developed solution (MIBK:IPA = 1:3) for $50 \mathrm{~s}$. In the following, 6-nm-thick titanium, 160-nm-thick $\mathrm{MgF}_{2}$ and 6-nm-thick titanium were deposited in sequence onto the sample. Furthermore, lift-off process was carried out by immersing the sample into the acetone solution for a day and then the beaker with the sample was put inside an ultrasonic vibrator for $2 \mathrm{~min}$ with a frequency of $80 \mathrm{kHz}$ and power efficiency of $40 \%$. The sample was then attached to a holder that tilted 86-degree with respect to the horizon in the e-beam evaporator with an operating pressure of $6 \times 10^{-6}$ torr. The reading from the quartz oscillator was $500 \mathrm{~nm}$ for aluminum deposition. Similar fabrication procedure was conducted for the planar MPA except that during deposition, the upper resonator was deposited continuously after the deposition of the seed layer.

Received: 12 October 2021; Accepted: 16 February 2022

Published online: 03 March 2022

\section{References}

1. Pendry, J. B. Negative refraction makes a perfect lens. Phys. Rev. Lett. 85, 3966-3969 (2000).

2. Smith, D. R., Padilla, W. J., Vier, D. C., Nemat-Nasser, S. C. \& Schultz, S. Composite medium with simultaneously negative permeability and permittivity. Phys. Rev. Lett. 84, 4184-4187 (2000).

3. Huang, T.-Y., Lin, J.-H., Lee, T. \& Yen, T.-J. Experimentally approaching to reciprocal cloaks via annulus-dielectric-metamaterials. J. Opt-uk 21, 08LT01 (2019).

4. Lin, J.-H., Yen, T.-J. \& Huang, T.-Y. Design of annulus-based dielectric metamaterial cloak with properties of illusion optics. J. Opt-uk 22, 085101 (2020).

5. Huang, T.-Y., Lai, Y.-C. \& Yen, T.-J. Slowing light by activating an oscillatory mode in a negative refractive waveguide composed of all dielectric metamaterials. Appl. Phys. Express 9, 012003 (2016).

6. Huang, T.-Y., Yang, T.-C. \& Yen, T.-J. Slowing light by exciting the fundamental degeneracy oscillatory mode in a negative refractive waveguide. Appl. Phys. Lett. 102, 111102 (2013).

7. Liu, C. et al. Slow light effect based on tunable plasmon-induced transparency of monolayer black phosphorus. J. Phys. D Appl. Phys. 52, 405203-405208 (2019).

8. Vafapour, Z. Polarization-independent perfect optical metamaterial absorber as a glucose sensor in food industry applications. IEEE Trans. Nanobiosci. 18, 622-627 (2019).

9. Naghdi, T. et al. Chitin nanofiber paper toward optical (bio)sensing applications. ACS Appl. Mater. Interfaces 12, 15538-15552 (2020).

10. Lin, C.-T., Yen, T.-J. \& Huang, T.-Y. PVC detection through a hybrid SEIRA substrate and refractive index sensor based on metamaterial perfect absorbers. Coatings 11, 789 (2021).

11. Pryce, I. M., Kelaita, Y. A., Aydin, K. \& Atwater, H. A. Compliant metamaterials for resonantly enhanced infrared absorption spectroscopy and refractive index sensing. ACS Nano 5, 8167-8174 (2011).

12. Lee, B.-S., Lin, D.-Z. \& Yen, T.-J. A low-cost, highly-stable surface enhanced Raman scattering substrate by Si nanowire arrays decorated with Au nanoparticles and Au backplate. Sci. Rep. 7, 4604 (2017).

13. Abdulkarim, Y. I. et al. The detection of chemical materials with a metamaterial-based sensor incorporating oval wing resonators. Electronics 9, 825 (2020).

14. Ma, R.-M., Ota, S., Li, Y., Yang, S. \& Zhang, X. Explosives detection in a lasing plasmon nanocavity.. Nat Nanotechnol. https://doi. org/10.1038/nnano.2014.135 (2014).

15. Liu, N., Mesch, M., Weiss, T., Hentschel, M. \& Giessen, H. Infrared perfect absorber and its application As plasmonic sensor. Nano Lett. 10, 2342-2348 (2010).

16. Gu, X. et al. Ultra-narrow-band perfect absorber based on high-order plasmonic resonance in metamaterial. J. Nonlinear Opt. Phys. 25, 1650011 (2016).

17. Van, P. C. et al. Broadband tunable plasmonic substrate using self-assembled gold-silver alloy nanoparticles. Curr. Appl. Phys. 19, 1245-1251 (2019).

18. Guo, L., Chen, L., Hong, S. \& Kim, D.-H. Single plasmonic nanoparticles for ultrasensitive DNA sensing: from invisible to visible. Biosens. Bioelectron. 79, 266-272 (2016).

19. Lee, K.-L., Wang, W.-S. \& Wei, P.-K. Sensitive label-free biosensors by using gap plasmons in gold nanoslits. Biosens. Bioelectron. 24, 210-215 (2008).

20. Wei, W., Zhang, X., Yu, H., Huang, Y. \& Ren, X. Plasmonic waveguiding properties of the gap plasmon mode with a dielectric substrate. Photonics Nanostruct. Fundam. Appl. 11, 279-287 (2013).

21. Ding, F., Yang, Y., Deshpande, R. A. \& Bozhevolnyi, S. I. A review of gap-surface plasmon metasurfaces: Fundamentals and applications. Nanophotonics-Berlin 7, 1129-1156 (2018).

22. Yong, Z., Zhang, S., Gong, C. \& He, S. Narrow band perfect absorber for maximum localized magnetic and electric field enhancement and sensing applications. Sci. Rep.-uk 6, 24063 (2016).

23. Emaminejad, H., Mir, A. \& Farmani, A. Design and simulation of a novel tunable terahertz biosensor based on metamaterials for simultaneous monitoring of blood and urine components. Plasmonics 16, 1537-1548 (2021).

24. Patel, S. K. \& Parmar, J. Highly sensitive and tunable refractive index biosensor based on phase change material. Phys. B Condens. Matter 622, 413357 (2021).

25. Wang, W., Yan, F., Tan, S., Zhou, H. \& Hou, Y. Ultrasensitive terahertz metamaterial sensor based on vertical split ring resonators. Photonics Res. 5, 571 (2017).

26. Yeh, T.-T., Huang, T.-Y., Tanaka, T. \& Yen, T.-J. Demonstration of a three-dimensional negative index medium operated at multipleangle incidences by monolithic metallic hemispherical shells. Sci. Rep. 7, 45549 (2017).

27. Chen, J. et al. Highly sensitive 3D metamaterial sensor based on diffraction coupling of magnetic plasmon resonances. Res. Phys. 15, 102791 (2019).

28. Yin, W., Shen, Z., Li, S., Zhang, L. \& Chen, X. A three-dimensional dual-band terahertz perfect absorber as a highly sensitive sensor. Aip Conf Proc 9, 665280 (2021).

29. Chen, J. et al. High sensing properties of magnetic plasmon resonance by strong coupling in three-dimensional metamaterials. J. Lightwave Technol. 39, 562-565 (2020).

30. Yang, J., Deng, H., Xiong, Z. \& Shang, L. Terahertz sensor based on a three-dimensional double I-type metamaterial integrated microfluidic channel. Appl. Opt. 60, 3816 (2021).

31. Zhu, J. et al. Three-dimensional cavity-coupled metamaterials for plasmonic color and real-time colorimetric biosensors. Nanoscale $12,4418-4425(2020)$.

32. Hu, X. et al. Metamaterial absorber integrated microfluidic terahertz sensors. Laser Photonics Rev. 10, 962-969 (2016). 
33. Geng, Z., Zhang, X., Fan, Z., Lv, X. \& Chen, H. A route to terahertz metamaterial biosensor integrated with microfluidics for liver cancer biomarker testing in early stage. Sci. Rep.-uk 7, 16378 (2017).

34. Zhou, R. et al. Label-free terahertz microfluidic biosensor for sensitive DNA detection using graphene-metasurface hybrid structures. Biosens. Bioelectron. 188, 113336 (2021).

35. Le, T. H. H. et al. Fabrication of infrared-compatible nanofluidic devices for plasmon-enhanced infrared absorption spectroscopy. Micromachines-Basel 11, 1062 (2020).

36. Le, T. H. H. \& Tanaka, T. Plasmonics-nanofluidics hydrid metamaterial: an ultrasensitive platform for infrared absorption spectroscopy and quantitative measurement of molecules. ACS Nano 11, 9780-9788 (2017).

37. Su, D.-S., Tsai, D. P., Yen, T.-J. \& Tanaka, T. ultrasensitive and selective gas sensor based on a channel plasmonic structure with an enormous hot spot region. Acs Sensors 4, 2900-2907 (2019).

38. Vick, D. et al. Self-shadowing and surface diffusion effects in obliquely deposited thin films. Thin Solid Films 339, 88-94 (1999).

39. Robbie, K. et al. Ultrahigh vacuum glancing angle deposition system for thin films with controlled three-dimensional nanoscale structure. Rev. Sci. Instrum. 75, 1089-1097 (2004).

40. Jensen, M. O. \& Brett, M. J. Periodically structured glancing angle deposition thin films. IEEE Trans. Nanotechnol. 4, 269-277 (2005).

41. Hawkeye, M. M. \& Brett, M. J. Glancing angle deposition: Fabrication, properties, and applications of micro- and nanostructured thin films. J. Vac. Sci. Technol. Vac. Surf. Films 25, 1317-1335 (2007).

42. Lu, C.-Y., Chung, C.-C., Yen, T.-J. \& Huang, T.-Y. Achieving broad absorption band and high incident angles by stochasticallydistributed oblique-flat-sheet metamaterial perfect absorbers. Sci. Rep.-UK 11, 18713 (2021).

43. Sun, J., Liu, L., Dong, G. \& Zhou, J. An extremely broad band metamaterial absorber based on destructive interference. Opt. Express 19, 21155 (2011)

44. Du, C. et al. An ultra-broadband terahertz metamaterial coherent absorber using multilayer electric ring resonator structures based on anti-reflection coating. Nanoscale 12, 9769-9775 (2020).

45. Vafapour, Z. Polarization-independent perfect optical metamaterial absorber as a glucose sensor in food industry applications. IEEE Trans. Nanobiosci. 18, 622-627 (2019).

46. Zhang, Z. et al. Mid-infrared sensor based on a suspended microracetrack resonator with lateral subwavelength-grating metamaterial cladding. IEEE Photonics J. 10, 1-8 (2018).

47. Akhavan, A., Ghafoorifard, H., Abdolhosseini, S. \& Habibiyan, H. Plasmon-induced transparency based on a triangle cavity coupled with an ellipse-ring resonator. Appl. Opt. 56, 9556 (2017).

48. Lu, X. et al. Numerical investigation of narrowband infrared absorber and sensor based on dielectric-metal metasurface. Opt. Express 26, 10179 (2018).

\section{Author contributions}

T.-Y. H. conceived the project, T.-Y. H., X.-X. W. and C.-Y. L. designed, simulated and measured the sample. T.-Y. H., and X.-X. W. co-wrote the paper. All authors discussed the results and commented on the manuscript.

\section{Funding}

This work is supported by the Ministry of Science and Technology (MOST 107-2218-E-131-005-MY3 and 110-2222-E-131-005).

\section{Competing interests}

The authors declare no competing interests.

\section{Additional information}

Supplementary Information The online version contains supplementary material available at https://doi.org/ 10.1038/s41598-022-07338-6.

Correspondence and requests for materials should be addressed to T.-Y.H.

Reprints and permissions information is available at www.nature.com/reprints.

Publisher's note Springer Nature remains neutral with regard to jurisdictional claims in published maps and institutional affiliations.

c) (i) Open Access This article is licensed under a Creative Commons Attribution 4.0 International (cc) License, which permits use, sharing, adaptation, distribution and reproduction in any medium or format, as long as you give appropriate credit to the original author(s) and the source, provide a link to the Creative Commons licence, and indicate if changes were made. The images or other third party material in this article are included in the article's Creative Commons licence, unless indicated otherwise in a credit line to the material. If material is not included in the article's Creative Commons licence and your intended use is not permitted by statutory regulation or exceeds the permitted use, you will need to obtain permission directly from the copyright holder. To view a copy of this licence, visit http://creativecommons.org/licenses/by/4.0/.

(c) The Author(s) 2022 